\title{
Clinical analysis of retinal detachment caused by ocular siderosis
}

\author{
Chao Feng \\ Aier Eye Hospital of Wuhan University \\ Chong Xu ( $\sim$ nitendo800@sina.com) \\ Xueqin Kang \\ Aier Eye Hospital of Wuhan University \\ Jianhua Wu \\ Aier Eye of Wuhan University
}

\section{Research article}

Keywords: Ocular siderosis, Retinal detachment, Retinal tears

Posted Date: February 27th, 2020

DOI: https://doi.org/10.21203/rs.2.24716/v1

License: (c) (i) This work is licensed under a Creative Commons Attribution 4.0 International License. Read Full License 


\begin{abstract}
Background: Until now, there was no report about retinal detachment caused by ocular siderosis. The aim of this study was to analyse the clinical characteristics of retinal detachment caused by ocular siderosis.
\end{abstract}

Methods: A retrospective cohort analysis was performed to analyse the clinical characteristics of retinal detachment caused by ocular siderosis in Aier Eye Hospital of Wuhan University from January 2016 to December 2019.

Results: There were 12 patients ( 12 eyes) with retinal detachment caused by ocular sclerosis, 11 males ( 11 eyes) and 1 female ( 1 eye), aged $20-54$ years. The median best corrected visual acuity (LogMAR) before and after surgery was 2.6 and 1.4, respectively. Retinal detachment recurred after the removal of silicone oil in four patients, all of whom had retinal tears.

Conclusion: The prognosis of retinal detachment caused by ocular siderosis is poor, and retinal detachment easily recurs when retinal tears exist.

\title{
Background
}

Ocular siderosis refers to a perforated eye injury in which iron foreign bodies enter the eye, persist and release iron ions for a long time,these foreign bodies spread via intraocular fluid flow, causing a series of characteristic changes that lead to severe visual dysfunction[1,2]. Retinal detachment is one of the many complications associated with ocular siderosis. In this paper, 12 cases of retinal detachment caused by ocular siderosis in Aier Eye Hospital of Wuhan University in the past 6 years were analysed retrospectively, and the aim of this study was to summarize and analyse the clinical characteristics of these patients.

\section{Methods}

The subjects were 12 patients (12 eyes) who were hospitalized in Aier Eye Hospital of Wuhan University from January 2016 to December 2019 and were clinically diagnosed with ocular siderosis complicated by retinal detachment. Inclusion criteria: (1) opacities of the lens and iron rust were observed via slitlamp examination[3], and orbital computed tomography (CT) scan or B-scan ultrasonography confirmed the presence of intraocular foreign bodies; and (2) Bscan ultrasonography confirmed retinal detachment. No obvious wounds were found on the ocular surfaces of the patients in this group. There were 11 males (11 eyes) and 1 female (1 eye), aged 20-64 years, with an average age of 39.5 years. The course of disease was 1-14 months, the mean course of disease was 6.42 months, the follow-up time was 7-19 months, and the mean follow-up time was 13.58 months.

\section{Surgery}

All patients underwent vitrectomy combined with foreign body removal, and cataract phacoemulsification was performed for those with obvious lens opacities that interfered with the surgical field of vision. The vitreous cavities of patients with exudative retinal detachment were filled with a balanced salt solution or sterile air after surgery. The vitreous cavity was tamponated with silicone oil after the operation for rhegmatogenous retinal detachment. The removal time of silicone oil was $3 \sim 6$ months after vitrectomy.

\section{Statistical analysis}

Snellen's visual acuity values were transformed into LogMAR visual acuity values. Non-Snellen vision values were recorded as follows: counting fingers was 2.6; hand motion was 2.7; and light projection was 2.8. Visual acuity was compared using the Wilcoxon rank sum test, and P<0.05 was considered statistically significant.

\section{Results}

\section{Best corrected visual acuity}

The preoperative best corrected visual acuities of all patients were 1.1-2.8, with a median of 2.6, and the postoperative best corrected visual acuities of all patients were 1.0-2.7, with a median of 1.4. Postoperative visual acuity improved in 6 cases ( 6 eyes) (50\%) and decreased in 1 case (1 eye) (8.33\%), and the other cases did not change $(41.67 \%)$. The difference between the best corrected visual acuity before and after surgery was statistically significant $(P=0.037)$.

\section{Recurrence Of Retinal Detachment}

In this group, there were 4 cases (4 eyes) of recurrent retinal detachment after the removal of silicone oil (33.33\%); all of these patients had retinal tears caused by foreign body injury to the retina. The recurrence time of retinal detachment was 1-3 weeks after the removal of silicone oil. All the patients with recurrent retinal detachment underwent reoperation, and the vitreous cavity was filled with silicone oil again. Among them, 2 patients ( 2 eyes) had recurrent retinal detachment after the secondary removal of silicone oil, and the other 2 patients (2 eyes) had not undergone removal of silicone oil within the follow-up period.

\section{Location Of The Foreign Body}


In this group, the location of the foreign body wasthe vitreous body cavity in 2 cases (16.67\%), ciliary body plane in 4 cases (33.33\%),peripheral part in 3 cases (25\%), equatorial part in 2 cases (16.67\%) and posterior pole in 1 case $(8.33 \%)$.

\section{Case}

Patient no. 6, a 33-year-old male patient, was admitted for 5 months with slow vision loss. Five months before seeking medical treatment, while interior decorating, the patient felt that there was a foreign body splashing and hitting the eye, and the discomfort in the eye disappeared after 1 day. However, the patient's vision had been declining since then, and he had not seen a doctor because of his busy work schedule. Eye condition at the first visit: The best corrected visual acuitywas HM, and no obvious wounds were found on the ocular surface. Thecorneal stroma was brown, the pupil was slightly dilated, and the diameter wasapproximately $5 \mathrm{~mm}$. The lens was obviously cloudy, and the surface of the anterior capsule was covered with brown pigment particles. The posterior segment of the eye was not visible. B-scan examination: vitreous opacity and retinal detachment. Orbital CT: there was a high possibility of metal foreign bodies in the eyeball. After complete preoperative preparation upon admission, surgical treatment was given. The blurred lens was removed by phacoemulsification, and the posterior capsule was retained. Whole retinal detachment was brown with extensive retinal vascular atresia. At 7 o 'clock, a metal foreign body was embedded in the eye wall in the peripheral part of the retina, and the retinal tear diameter was approximately 0.5 PD. The foreign body was removed, and the retina was reattached via three-channel vitrectomy. Laser photocoagulation was performed at the edge of the retinal tear (laser spot number:75 points), and silicone oil was used to fill the vitreous cavity before the operation was completed.

There was no significant improvement in the patient's best corrected visual acuity after regular follow-up, but the retina was well repositioned.The silicone oil was removed 3 months after the first operation. During the second operation, it was found that the retina wascompletely fitted, and there was no obvious proliferative vitreoretinopathy, but retinal vascular filling was still insufficient, the laser spot at the retinal tear edge was not clear, and the vitreous cavity was filled with balanced salt solution. Unfortunately, one week after the removal of silicone oil, the patient experienced the first recurrence of retinal detachment and received a third surgical treatment. The patient'svitreous cavity was filled with silicone oil for the second time after the retina was repositioned again. At the 6th month after the third operation, no significant retinal detachment was observed in the eye, and the patient was required to undergo removal of the silicone oil. Therefore, the patient underwent a fourth operation. The silicone oil was removed from the eye for the second time. During this operation, the retina was still completely occluded, and no obvious proliferative vitreoretinopathy was observed. Then, the eye was filled with balanced salt solution again. At the third week of follow-up after the fourth operation, the patient had a second recurrence of retinal detachment. Doctors had no choice but to give the patient a fifth operation to reposition the retina and a third implementation of tamponated silicone oil. The patient is still in follow-up, and the retina is well restored under the surface tension of silicone oil, but the best corrected visual acuity is not significantly improved compared with that before the first operation.

\section{Discussion}

Ocular siderosis includes a series of degenerative changes caused by iron poisoning in the eye, such as keratopathy, heterochromia iridum, tetanicpupils, dilated pupils, secondary glaucoma, and complicated cataracts[3-5]. The complications in the posterior segment of the eye caused by ocular siderosis include cystic macular oedema, diffuse pigment changes, arteriole stenosis, retinal ischaemia, retinal detachment and severe proliferative vitreoretinopathy[6]. The causes of retinal detachment due toocular siderosis are retinal glial cell proliferation and subretinal leakage[7]. Until now, there have been no reports about retinal detachment caused by ocular siderosis.

This study is the first to observe the clinical characteristics of retinal detachment caused by ocular siderosis. The clinical characteristics of this group of patients can be summarized as follows: 1. the location of foreign body deposition is relatively hidden; 2. poor vision prognosis; and 3. retinal detachment is highly likely to recur in patients with retinal tears. There were 7 cases ( 7 eyes), accounting for $58.33 \%$, in which foreign bodies were located in the plane part of the ciliary body or the peripheral part of the retina in this group. Because the location of foreign body deposition is relatively hidden and the size of iron foreign bodiesis usually small, the early clinical symptoms of patients with injuries are often not obvious, and the presence of foreign bodies is difficult to identify by routine examination, so the course of disease is prone to be delayed.Failure to seek early treatment is also an important practical cause ofocular siderosis with retinal detachment.

Although the exact mechanism by which iron causes widespread retinal degeneration and vascular lesions is unclear, many hypotheses exist. One hypothesis is that ions released by iron produce free radicals that cause severe oxidative damage to retinal cells $[6,8,9]$. The Haber-Weiss reaction, in which $\mathrm{Fe}^{3+}$ and $\mathrm{O}_{2}{ }^{-}$ are converted to strong oxidizing hydroxyl radicals, is thought to occur in vivo, as these reactants may persist for a long time in the case of iron deposition. $\mathrm{Fe}^{3+}$ is the catalyst in the reaction, which means it is present as a reactant in the first step and is regenerated in the last step, so only a small amount of Fe ${ }^{3+}$ can push the reaction forward indefinitely. While $\mathrm{O}_{2}{ }^{-}$is produced by light and molecular oxygen, long-term exposure to the strong oxidative environment of superoxide dismutase and catalase cannot remove or neutralize the harmful chemicals in these cells[6]. Another hypothesis, based on the cytotoxic mechanisms of silicosis and asbestosis, postulates that the accumulation of iron in cells leads to the release of lysosomal enzymes, which damage tissues[8]. This hypothesis suggests that cytoplasmic aggregates of ferritin, known as ferriosomes, occur in ocular iron deposition, triggering a toxic cascade. Therefore, patients with ocular siderosis complicatedby retinal detachment tend to have poor vision, and the improvement of visual function is extremely limited even after surgical treatment. Moreover, even after the removal of intraocular foreign bodies, the visual function of some patients continues to decline. The possible reason is that the residual iron ions still promote intraocular toxicity even after the removal of intraocular foreign bodies. Small iron particles can still be released at the inner retinal surface following surgical removal, potentially inducing further toxicity[10, 11]and leading to further damage to retinal cells, resulting in the continuous decline of visual function. The overall postoperative visual acuity of all the patients in this group was poor, but the statistical results showed that the visual acuity of the patients after surgery was still significantly improved compared with that before surgery $(P<0.05)$, which may indicate that the surgical treatment of retinal detachment caused by ocular siderosis is still of clinical significance. 
Amongcases of retinal detachment caused by ocular siderosis, there is a special case whereexudative retinal detachment is accompanied by retinal tears caused by foreign bodies. In this study, 4 patients ( 4 eyes) had retinal tears. All 4 patients underwent filling with silicone oil during the first operation, and silicone oil was removed within 3 to 6 months after vitrectomy. Unfortunately, 4 of the patients experienced retinal detachment recurrence within 1 to 3 weeks after the removal of silicone oil. All of the patients with recurrent retinal detachment were subsequently treated with a second retinal reattachment, and the eyes were filled with silicone oil for the second time. Among them, two patients underwent silicone oil removal again within 6 to 12 months after the second retinopathy surgery, and retinal detachment recurrence occurred again, while the other two patients who underwent filling with silicone oil for a second time did not undergo silicone oil removal during the follow-up period. These results may suggest that patients with ocular siderosis complicated by rhegmatogenous retinal detachment have a low rate of complete retinal reattachment and are highlylikely to develop silicone oil-dependent eyes. The reason may be that iron ions tend to bind to retinal pigment epithelial cells, resulting in degeneration and inactivation of these cells[12]. Iron retinotoxicity leads to dysfunction of all the layers of the retina, with more severe damage occurring in the inner retina than in the outer retina in the late stages of the disease[8, 11],thus,effective and strong adhesion is difficult to form between the retinal neuroepithelium and pigment epithelium. This results in retinal tears not being completely closed even with retinal laser photocoagulation or retinal freezing. When the silicone oil in the eye is removed, the retinal tear can easily reopen, which leads to retinal detachmentrecurrence. When silicone oil is removed from the eye, the retinal tear tends to reopen, leading to retinal detachmentrecurrence. Further, the eyeballs of patients with ocular siderosis complicated by rhegmatogenous retinal detachment likely become silicone-oil-dependent eyes. Animal studies of ocular siderosis have also shown that the retina will completely degenerate, and degeneration of the pigment epithelium can cause the retina to fail to fuse with the choroid in the late stage of the disease[6]. Therefore, ocular siderosis complicated by rhegmatogenous retinal detachment is a special type of disease thathas a different prognosis from thatof exudative retinal detachment caused by ocular siderosis. Certainly, if iatrogenic retinal tears occur during surgical procedures for exudative retinal detachment due to ocular siderosis, the prognosis is the same. Full awareness and communication with the patient should be completed before the surgeon performs surgical treatment so that the patient can fully understand the severity of the disease.

This study is limited by its retrospective design. Furthermore, the sample size of this study is small, which does not fully reflect the overall situation of the disease. This study is only a single group of case cohort observations.

\section{Conclusions}

In summary, the vision prognosis of patients with retinal detachment caused by ocular siderosis is poor, and thesepatients are prone to relapse and the formation of silicone-oil-dependent eyes when combined with retinal tears.

\section{Declarations}

\section{Acknowledgments}

None.

\section{Authors' contributions}

CF, CX and JW designed and drafted the work, CF and CX contributed to the conception, CF, CX, XK, and JW interpreted the data, and XK acquired the data. All authors have read and approved the manuscript in its current state.

\section{Funding}

This work was supported by Wuhan Municipal Health and Family Planning Research Fund (WX18Q24), Wuhan Health Research Fund (WX19Q39) and the Scientific Research Fund of Aier Eye Hospital Group (AF1901D5).

\section{Availability of data and materials}

The data used to support the findings of this study are included within the article.

\section{Ethics approval and consent to participate}

We conducted this study was conducted in accordance with the Declaration of Helsinki, and ethical approval was obtained from the ethics committee of Aier Eye Hospital of Wuhan University.

\section{Consent for publication}

We obtained written informed consent from all participating patients for the publication.

\section{Competing interests}

The authors declare that they have no competing interests.

\section{References}

1. Burch PG, Albert DM: Transscleral ocular siderosis. Am J Ophthalmo/1977,84(1):90-97. 
2. Schechner R, Miller B, Merksamer E, Perlman I: A long term follow up of ocular siderosis: quantitative assessment of the electroretinogram. Doc Ophthalmo/1990, 76(3):231-240.

3. Weiss MJ, Hofeldt AJ, Behrens M, Fisher K: Ocular siderosis. Diagnosis and management. Retina 1997, 17(2):105-108.

4. Sneed SR, Weingeist TA: Management of siderosis bulbi due to a retained iron-containing intraocular foreign body. Ophthalmology 1990, 97(3):375-379.

5. Sangermani C, Mora P, Mancini C, Vecchi M, Gandolfi SA: Ultrasound biomicroscopy in two cases of ocular siderosis with secondary glaucoma. Acta Ophthalmol2010, 88(1):e1-e2.

6. Sandhu HS, Young LH: Ocular siderosis. Int Ophthalmol Clin 2013, 53(4):177-184.

7. Xie H, Chen S: Ocular siderosis. Eye Sci 2013, 28(2):108-112.

8. Imaizumi M, Matsumoto CS, Yamada K, Nanba Y, Takaki Y, Nakatsuka K: Electroretinographic assessment of early changes in ocular siderosis. Ophthalmologica2000, 214(5):354-359.

9. Kannan NB, Adenuga OO, Rajan RP, Ramasamy K: Management of Ocular Siderosis: Visual Outcome and Electroretinographic Changes. J Ophthalmo/2016, 2016:7272465-7272465.

10. Pollack A, Oliver M: Reversal of siderosis. Arch Ophthalmo/1998, 116(5):678-679.

11. Faure C, Gocho K, Le Mer Y, Sahel JA, Paques M, Audo I: Functional and high resolution retinal imaging assessment in a case of ocular siderosis. Doc Ophthalmol2014, 128(1):69-75.

12. Tawara A: Transformation and cytotoxicity of iron in siderosis bulbi. Invest Ophthalmol Vis Sci 1986, 27(2):226-236.

\section{Table}

\begin{tabular}{|c|c|c|c|c|c|c|c|c|c|c|}
\hline Patient & $\begin{array}{l}\text { Age } \\
\text { (year) }\end{array}$ & $\begin{array}{l}\text { Course } \\
\text { of } \\
\text { disease } \\
\text { (month) }\end{array}$ & $\begin{array}{l}\text { Location } \\
\text { of foreign } \\
\text { body }\end{array}$ & $\begin{array}{l}\text { whether } \\
\text { retinal } \\
\text { tear } \\
\text { exist }\end{array}$ & Operation & $\begin{array}{l}\text { Retinal } \\
\text { detachment } \\
\text { recurred }\end{array}$ & $\begin{array}{l}\text { Time of } \\
\text { retinal } \\
\text { detachment } \\
\text { recurrence } \\
\text { (week) }\end{array}$ & $\begin{array}{l}\text { Preoperative } \\
\text { VA }\end{array}$ & $\begin{array}{l}\text { - Postoperative } \\
\text { VA }\end{array}$ & $\begin{array}{l}\text { Foll } \\
\text { up } \\
\text { (mc }\end{array}$ \\
\hline 1 & 53 & 12 & $\begin{array}{l}3 \text { clock, } \\
\text { ciliary } \\
\text { pars } \\
\text { plana }\end{array}$ & no & Phaco+PPV+IOFBR & no & / & $\mathrm{LP} \rrbracket 2.8 \rrbracket$ & $0.08 \otimes 1.1 \rrbracket$ & 14 \\
\hline 2 & 64 & 7 & $\begin{array}{l}5 \text { clock, } \\
\text { equator }\end{array}$ & yes & Phaco+PPV+IOFBR+SOT & yes & 1 & $\mathrm{CF} \rrbracket 2.6 \rrbracket$ & $\mathrm{HM} \otimes 2.7 \rrbracket$ & 19 \\
\hline 3 & 25 & 6 & $\begin{array}{l}4 \text { clock, } \\
\text { ciliary } \\
\text { pars } \\
\text { plana }\end{array}$ & no & Phaco+PPV+ IOFBR & no & / & $0.04 \bigotimes 1.4 \rrbracket$ & $0.04 \rrbracket 1.4 \rrbracket$ & 7 \\
\hline 4 & 49 & 14 & $\begin{array}{l}5 \text { clock, } \\
\text { periretinal }\end{array}$ & no & Phaco+PPV+ IOFBR & no & / & CF囚2.6囚 & $0.04 \rrbracket 1.4 \rrbracket$ & 12 \\
\hline 5 & 29 & 1 & $\begin{array}{l}\text { Inferior, } \\
\text { vitreous } \\
\text { cavity }\end{array}$ & no & PPV+ IOFBR & no & / & $0.08 \otimes 1.1 \rrbracket$ & $0.12 \bowtie 0.9 \rrbracket$ & 10 \\
\hline $6^{*}$ & 33 & 5 & $\begin{array}{l}7 \text { clock, } \\
\text { periretinal }\end{array}$ & yes & $\begin{array}{l}\text { Phaco+PPV+ IOFBR } \\
\text { +SOT }\end{array}$ & yes & 2 & $\mathrm{HM} \unrhd 2.7 \rrbracket$ & $\mathrm{HM} \otimes 2.7 \rrbracket$ & 15 \\
\hline 7 & 28 & 2 & $\begin{array}{l}\text { Inferior, } \\
\text { vitreous } \\
\text { cavity }\end{array}$ & no & PPV+ IOFBR & no & / & $0.02 \bigotimes 1.7 \rrbracket$ & $0.08 \otimes 1.1 \rrbracket$ & 18 \\
\hline $8^{*}$ & 38 & 6 & $\begin{array}{l}4 \text { clock, } \\
\text { equator }\end{array}$ & yes & $\begin{array}{l}\text { Phaco+PPV+ } \\
\text { IOFBR+SOT }\end{array}$ & yes & 3 & $\mathrm{CF} \otimes 2.6 \rrbracket$ & $\mathrm{CF} \otimes 2.6 \rrbracket$ & 16 \\
\hline 9 & 43 & 4 & $\begin{array}{l}7 \text { clock, } \\
\text { ciliary } \\
\text { pars } \\
\text { plana }\end{array}$ & no & Phaco+PPV+ IOFBR & no & / & $0.02 \otimes 1.7 \rrbracket$ & $0.02 \rrbracket 1.7 \rrbracket$ & 12 \\
\hline 10 & 48 & 10 & $\begin{array}{l}\text { Inferior } \\
\text { temporal } \\
\text { near optic } \\
\text { disc }\end{array}$ & yes & $\begin{array}{l}\text { Phaco+PPV+ } \\
\text { IOFBR+SOT }\end{array}$ & yes & 1 & LP冈2.8囚 & $\mathrm{LP} \otimes 2.8 \rrbracket$ & 13 \\
\hline 11 & 44 & 8 & $\begin{array}{l}9 \text { clock, } \\
\text { periretinal }\end{array}$ & no & Phaco+PPV+ IOFBR & no & / & $\mathrm{HM} \otimes 2.7 ه$ & $0.06 \rrbracket 1.2 \rrbracket$ & 15 \\
\hline 12 & 20 & 2 & $\begin{array}{l}3 \text { clock, } \\
\text { ciliary } \\
\text { pars } \\
\text { plana }\end{array}$ & no & PPV+ IOFBR & no & / & $0.02 \bigotimes 1.7 \rrbracket$ & $0.1 \otimes 1.0 \bigotimes$ & 12 \\
\hline
\end{tabular}


Phaco: Phacoemulsification; PPV: Pars Plana Vitrectomy; IOFBR: Intraocular Foreign Body Removal; SOT Silicone Oil Tamponate; VA: Visual Acuity; CF: Counting Fingers; HM: Hand Motion; LP: Light Projection; *: The patient experienced two relapses of retinal detachment 